\title{
Des chars à bœufs aux plateformes mobiles de
} forage

Réparation, fabrication et innovation à Tiruchengode

\section{Yann Philippe Tastevin}

\section{OpenEdition}

\section{Journals}

Édition électronique

URL : https://journals.openedition.org/tc/8488

DOI : $10.4000 /$ tc. 8488

ISSN : $1952-420 X$

\section{Éditeur}

Éditions de l'EHESS

Édition imprimée

Date de publication : 6 juin 2017

Pagination : 196-211

ISBN : 978-2-7132-2707-3

ISSN : 0248-6016

\section{Référence électronique}

Yann Philippe Tastevin, «Des chars à bœufs aux plateformes mobiles de forage », Techniques \&

Culture [En ligne], 67 | 2017, mis en ligne le 06 juin 2019, consulté le 29 septembre 2022. URL : http:// journals.openedition.org/tc/8488; DOI : https://doi.org/10.4000/tc.8488 


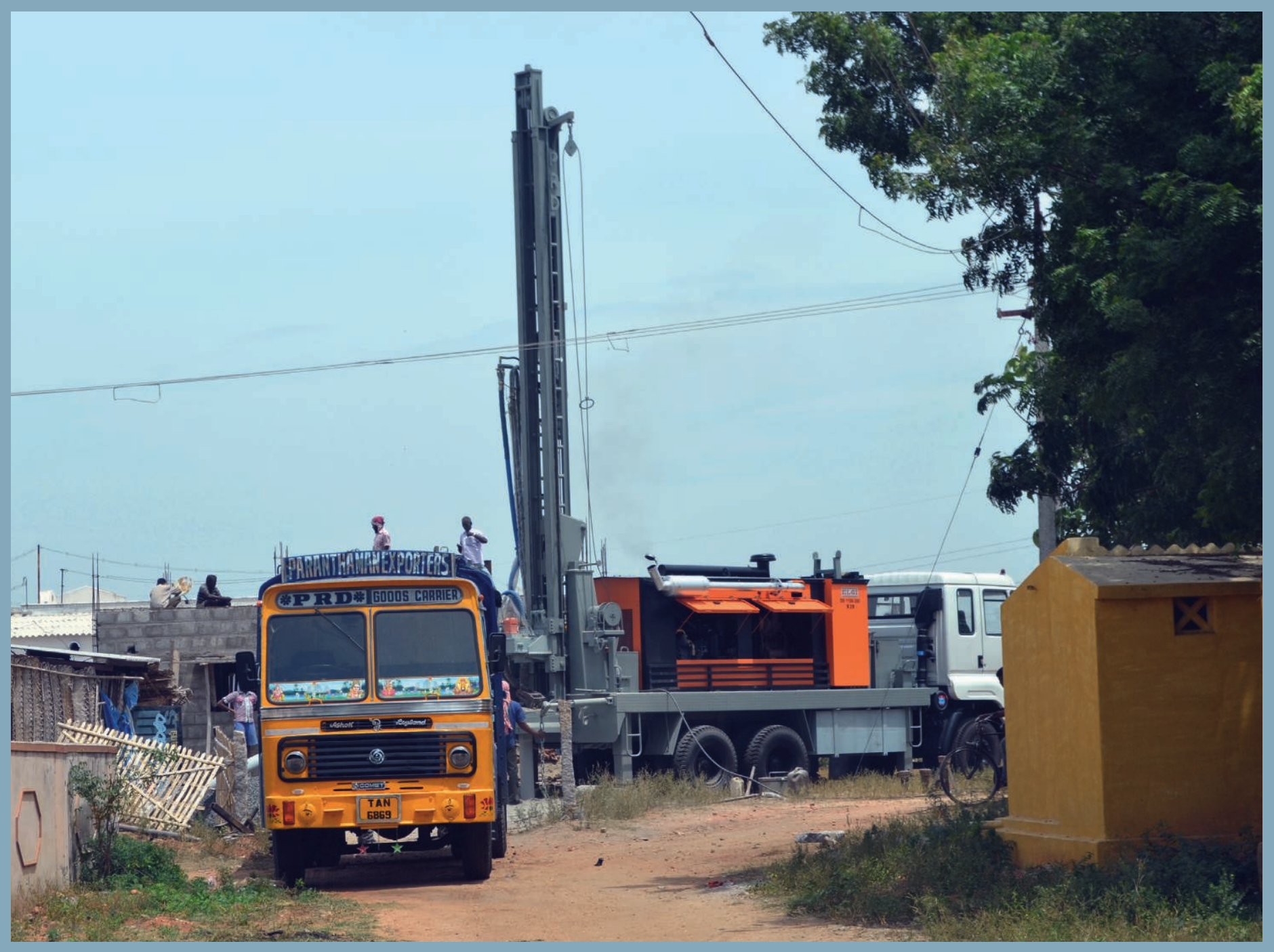




\section{Des chars à bœufs aux plateformes mobiles de forage} Réparation, fabrication et innovation à Tiruchengode

Tiruchengode, située dans le district de Salem sur le plateau du Carnatic au Tamil Nadu en Inde du Sud est une localité signalée dans de nombreux travaux qui s'interrogent sur les relations entre urbanisation et industrialisation (Cadène 1991, Cadène \& Holmström 1998, Kundu 1994, Roda 1994, Raman 2013). Dans cette ville, une foule de petites entreprises ont développé une activité productive et commerciale intense dans le domaine du textile, mais aussi de manière plus surprenante dans le transport puis le forage de puits d'eau. Aujourd'hui, ces deux activités dynamisent une agglomération forte de ses 95000 habitants en 2011, et leurs filières rayonnent à l'échelle du pays et s'ouvrent à l'international.

En détaillant l'histoire d'une spécialisation urbaine de l'entretien de la réparation et du carrossage de camions (lorry body-building) puis de l'assemblage de plateformes mobiles de forage (mobile drilling rigs), cet article montre comment l'innovation technique s'appuie à Tiruchengode sur le retournement du cycle classique - besoin, design, implémentation, vérification, maintenance. La maintenance y oriente la conception, génère un marché et joue de la réinvention ordinaire des choses. Si l'histoire des techniques du xxe siècle abonde en exemples d'entreprises qui débutent par l'entretien d'une technologie et passent à la fabrication de composants ou de l'objet entier, et enfin à l'innovation ${ }^{1}$, cette contribution explore à Tiruchengode un processus d'adaptation par rétro-ingénierie (reverse engineering), où l'innovation s'immisce dans les chaînes de duplication artisanale d'une technologie venue d'ailleurs. Le déploiement d'un cluster d'assemblage de rigs (plateformes) a donné naissance à une firme transnationale, là où on ne l'attendait pas. Le cas de cette entreprise familiale est unique à Tiruchengode par son ancienneté et désormais par sa taille. PRD se différencie non seulement par sa propension, depuis une décennie à explorer des marchés outremer (Kenya, Ghana, Éthiopie...), mais surtout par sa capacité à refaire toute sorte de machines. 


\section{Entretien et trouvailles}

Située dans une région cotonnière ${ }^{2}$, Tiruchengode est, historiquement, une ville de tisserands mais aussi de forgerons, où les métiers à tisser et les chars à bœufs prospèrent depuis l'indépendance et font la réputation du lieu. Tandis que la mécanisation du tissage induit la qualification « sur le tas » de la main-d'œuvre, certains ouvriers se spécialisent dans le fonctionnement de machines nouvelles. Cette maintenance ad hoc génère une culture technique locale (Kundu 1994) qui facilite les transferts. Savoir-faire et connaissances techniques proviennent de l'artisanat des métaux traditionnels et des premiers ateliers de réparation des métiers à tisser. Entre la ville, le centre de l'activité et les différents travailleurs qui élaborent le textile, les systèmes de transport se développent sur de courtes distances. Avec l'arrivée des premiers camions, la motorisation devient une opportunité de diversification de la fabrique locale où se développent les compétences et s'enchevêtrent les activités. Parallèlement à la fabrication de chars à bœufs, les ateliers polyvalents de forgerons se spécialisent dans la réparation automobile (le réseau routier se développe alors rapidement). Le constructeur anglais Leyland en partenariat avec Ashok Motor installe les premières chaînes d'assemblage de châssis de camions à Ennode au sud de Madras. Le Comet 350 parmi les plus légers de la gamme routière constitue les premières flottes de camions et d'autobus (Bedford SB) qui se répandent dans la région. Certains commerçants deviennent des transporteurs, et, dans le sillon des premiers véhicules motorisés, un milieu de petits patrons artisans et d'ouvriers mécaniciens se constitue à partir d'échoppes et d'ateliers où l'on entretient tout ce qui roule. Localement, les artisans se familiarisent à diverses techniques venues d'ailleurs tout en ayant à peine les moyens

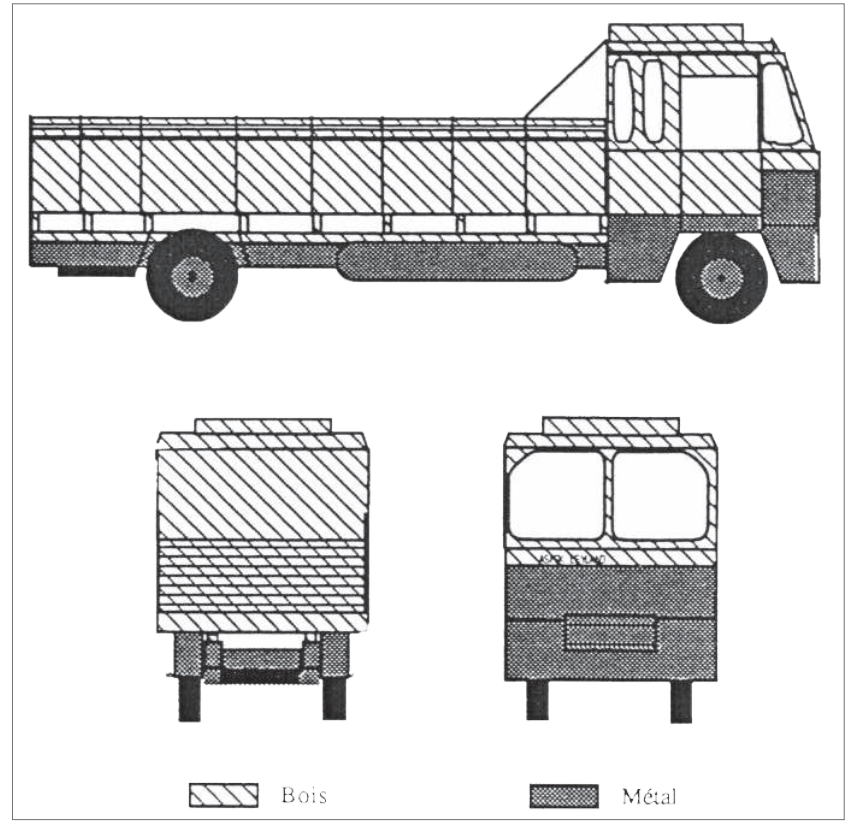

de les mettre en œuvre. Proposant un service aux pionniers du transport terrestre, les forgerons pour qui le travail des métaux et l'assemblage de chars en bois n'ont pas de secret, réparent désormais les éléments en bois et les parties métalliques de la cabine et de la plateforme arrière qui composent un camion. En les restaurant, les artisans réparateurs observent la conception des carrosseries. Pour un camion, la structure est beaucoup plus grande et ses composants en bois beaucoup plus lourds qu'un char mais l'assemblage du tout reste rudimentaire.

La réparation sur place de véhicules importés préfigure le développement d'une industrie de carrossage et d'usinage (Cadène 1991 op. cit., Tastevin 2017). De jeunes artisans héritiers de la forge ou de la charpente se spécialisent dans l'entretien et la réparation des camions anglais en réalisant de petites pièces simples. À l'assemblage " grossier» de poutres, de planches, de panneaux de bois et de montants en acier qui composent le carrossage, certains comme Thaman préfèrent 
l'usinage de composants mécaniques de plus en plus compliqués. Le façonnage de pièces de rechange exige de nouvelles machines-outils : perceuse à colonne, affûteuse, fraiseuse apparaissent avec l'électrification de la ville à la fin des années 1960. Thaman, fils de forgeron, se procure le premier un tour à métaux électrique d'occasion, un appareil capable entre ses mains expertes de transformer des blocs de métal ou d'aluminium en pièces mécaniques de précision. À force de couper, percer, limer, il peut transformer ces blocs de fonte ou d'acier pour en extraire un piston, des coussinets et des soupapes. D'une masse métallique, il fait une machine de précision. Thaman devient une mini-usine, capable de

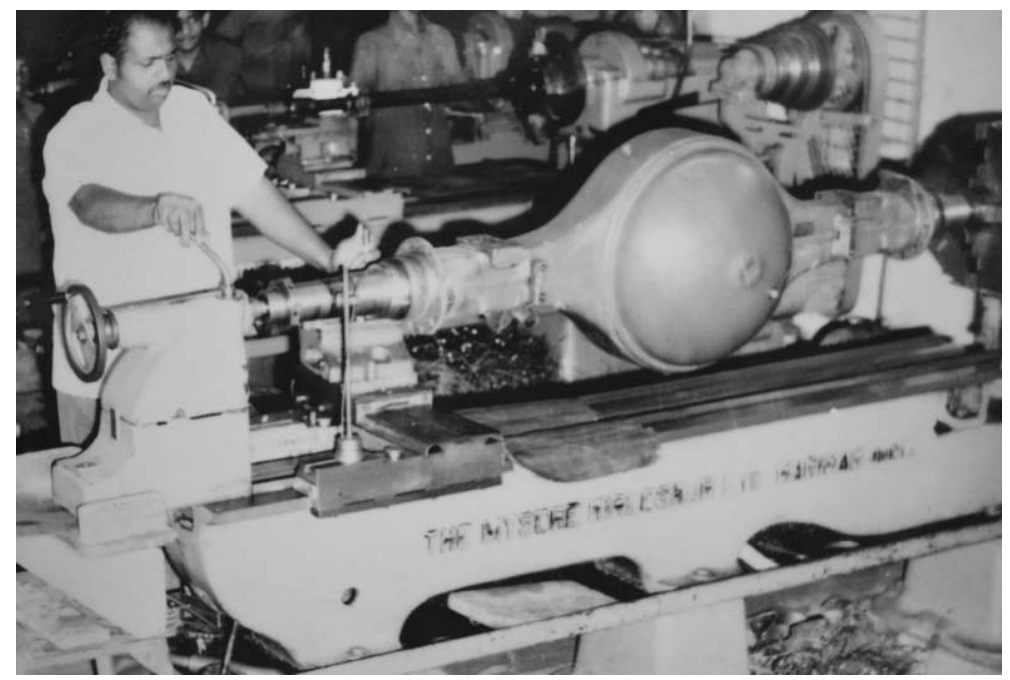
fabriquer toute sorte de pièces mécaniques.

Il devient expert dans le dépannage du pont moteur des camions Ashok Leyland ${ }^{3}$, il répare 2. Pont et différentiel les essieux arrière qui résistent mal au chargement de néotransporteurs qui sillonnent des routes fraîchement bitumées de la région en maximisant la cargaison : «In England, Leyland was manufacturing for 6 tons capacity trucks. When they came to India, they assembled the same model. But the Indians transporters overloaded the lorry that is why it breaks ${ }^{4}$. » Cette panne récurrente ralentit le roulage de marchandises, le plus souvent périssables, et coûte cher aux transporteurs. La charge à l'essieu pratiquée en Inde incite le réparateur à adapter le système de suspension arrière $^{5}$. Comme l'assemblage du pont est un agencement simple et rigide, il peut être renforcé. En démontant systématiquement les éléments suspendus par des ressorts à lames, soit un différentiel inséré dans un carter unique contenant les arbres de transmission reliant le différentiel aux roues motrices, Thaman comprend l'enchaînement à l'origine du bris mécanique et trouve le moyen de consolider l'ensemble en rectifiant la petite pièce qui casse. Il met au point non pas une nouvelle pièce mais corrige un défaut de conception, il introduit une soudure. Cette amélioration technique ( mechanical fix») est si efficace qu'elle fait sa réputation : «by fixing the axle of the Ashok Leyland truck, my name became famous in all Tamil Nadu ${ }^{6}$. »

Petits patrons d'ateliers, artisans, transporteurs, reconnaissent l'habileté du fondateur de PRD qui deviendra principal constructeur du district. Alors que le fabricant de châssis roulant n'offrait aucune garantie à l'achat, le tourneur de Tiruchengode (lathe man) décide de promouvoir sa solution en garantissant la réparation pendant deux ans. L'information circule parmi les transporteurs de la région et les principaux clients d'Ashok Leyland avertissent ses distributeurs agréés. Si la promotion de cette révision et surtout sa garantie donnent lieu à un contentieux avec le constructeur, cette assurance artisanale fait la fortune de Thaman. Elle fidélise les transporteurs de la région et révèle au constructeur l'importance d'un espace discret de la maintenance. 
Dans le district, les artisans s'organisent. D'anciens chauffeurs s'installent, des ouvriers s'établissent à leur compte, la main-d'œuvre qualifiée circule d'atelier en atelier, les compétences et les trouvailles se transmettent, l'argent s'accumule. Les premières associations naissent pour défendre collectivement les intérêts d'une corporation en pleine expansion ${ }^{7}$. L'expérience collective constitue un capital social et technique, qui s'attache à l'entretien et à la réparation de toute sorte de matériels roulants. Adaptés à la taille des châssis poids lourds, les garages de la zone (motor workshop) se spécialisent dans la réparation, la rénovation (ou parfois la reconstruction) de véhicules automobiles de fort tonnage, destinés au transport des marchandises (camions) et des personnes (autocars). Les carrossiers sont reconnus dans tout le pays. La réparation de véhicule, la transformation d'utilitaires importés (Ford Transit (1953-) ou de camionnettes Bedford équipées alors de moteurs Perkins 4 cylindres $^{8}$ ) puis la préparation des châssis Ashok Leyland immobilisent sur place une main-d'œuvre qualifiée qui donne naissance à une forme de production répartie, complémentaire d'usines centralisées, car plus flexibles et capables de produire en plus petits lots. Habiller ou reconstruire le châssis d'un camion mobilise une trentaine de travailleurs qualifiés (soudure, menuiserie, électricité, peinture). Les métiers et les équipes interviennent successivement et simultanément sur le châssis (Cadène \& Holmström op. cit.), de sorte qu'artisans, entrepreneurs et commerçants de Tiruchengode forment une communauté de pratiques où savoir-faire et informations commerciales circulent. Le district périphérique par son dynamisme et ses réseaux de fournisseurs et d'acheteurs s'agglomère aux chaînes logistiques de centres industriels des états voisins. C'est ainsi qu'il attire et capte tout ce qui roule et finalement tombe en panne.

\section{De la réparation à la fabrication}

La mémoire de l'apparition de camions bardés d'une machine pneumatique de forage reste vive parmi les artisans de Tiruchengode. D'énormes engins avec leur mât et leurs foreuses sillonnaient les campagnes avoisinantes où les matériels de sondage vertical commençaient seulement à être utilisés pour explorer les sols et trouver de l'eau. Les technologies pneumatiques de forage et de construction, les compresseurs à air comprimé, leurs groupes électrogènes arrivaient alors des États-Unis (Chicago Pneumatique), de Suède (Atlas Corpo) ou d'Allemagne (Uraca). Irrigation, construction des routes, exploitation minière, la modernisation planifiée de l'Inde nécessitait de nouvelles machines, Nehru (1947-1964) multipliait les visites d'usines à l'étranger et les accords avec les fabricants de machines-outils. Les premiers ateliers de montage se sont implantés à la fin des années 1960 à Hyderabad, qui reste aujourd'hui encore, avec Bangalore, l'un des principaux centres de production de rigs.

En réparant les choses qui viennent d'ailleurs, l'industrie artisanale de Tiruchengode accumule collectivement l'état d'esprit et des compétences essentielles à la fabrication des objets. En l'occurrence un appareil mobile de forage pneumatique : 
"Someone came and asked him, could you make a rig. Just like that. As a kind of reverse engineer, he started to do his first rig. He was happy to make his first machine [...]. In that time he just made one machine, it wasn't perfect but it works. The government was importing the U.S. drilling technology, and some private company from Hyderabad started to import and then to assemble the rigs locally. In Tiruchengode, business as usual started with the maintenance and repair of the new technology: the American genuine and the duplicated one. It took two months of an intensive and obsessive work to learn and develop by doing the first imperfect rig made in Tiruchengode 9 . »

Pour concevoir son prototype ou refaire un rig, Thaman se rend à Hyderabad dans les « usines à ciel ouvert » pour observer le mode de construction : un processus d'assemblage plutôt que de fabrication. Comme à Tiruchengode, il n'y a pas de chaîne de montage à proprement dit, mais de grands garages dans lesquels on prépare puis assemble les plateformes. Thaman y rencontre les sous-traitants, mais aussi les exploitants des chantiers de forages. Ils discutent des avantages et des problèmes des nouvelles machines. Il y trouve aussi les principaux équipementiers étrangers qui fournissent les composants spécifiques dont la pièce maîtresse pour forer : le compresseur. La plus chère aussi. Ainsi pour l'acquérir ainsi que le reste des systèmes d'alimentation, de transmission mécanique, de rotation (rotary), tiges et outils de forages (rods \& bits), il a besoin d'argent. Et pour assembler le tout, il a besoin d'espace. La transformation de l'atelier mécanique pour adapter la nouvelle technologie nécessite de gros investissements, du capital. Pour la première fois, il doit s'endetter auprès de ses proches. En 1982, il fonde sa deuxième entreprise, Paranthaman Rock Drill (« the workshop became a company: PRD »). Thaman cesse alors l'entretien et la réparation de camion pour produire des borewell rigs. La préparation des châssis sur lesquels sont montées de lourdes plateformes de forage capables de supporter un mât de forage et des compresseurs exige un ensemble de techniques d'assemblage de pièces mécaniques par soudage, rivetage, boulonnage... Les longerons du châssis d'origine sont renforcés par le boulonnage de deux longerons supplémentaires pour supporter le poids de la plateforme. Ladaptation du châssis consiste ensuite à libérer de la place pour les éléments hydrauliques de la plateforme comme la pompe. Le réservoir d'origine est déplacé et remplacé par un autre de plus grande capacité (cf. photos 4 à 9) (Cadène op. cit., Tastevin op. cit.) ${ }^{10}$.

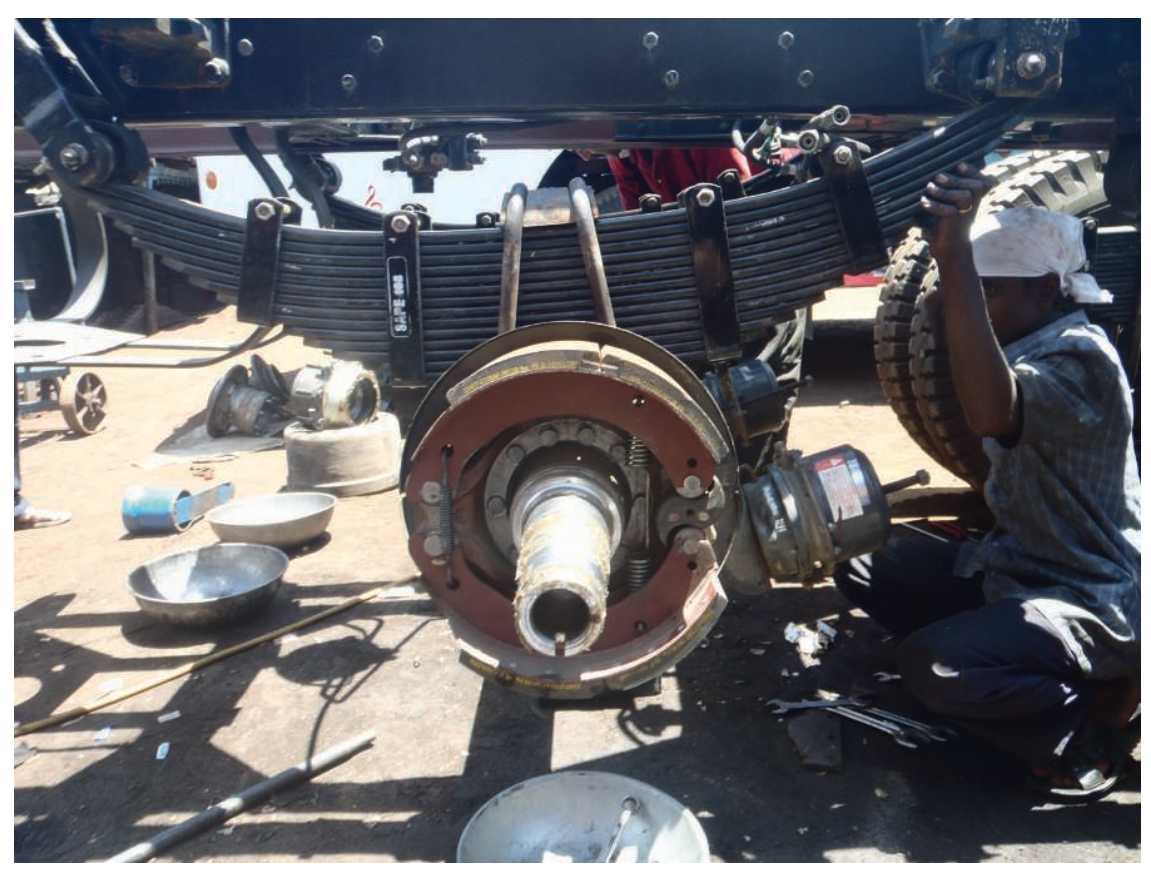


4 à 9. Préparation des châssis sur lesquels sont montées de lourdes plateformes capables de supporter un mât de forage et des compresseurs
À Tiruchengode, l'urbanisation in situ démultiplie les besoins en eau. Si, l'exploitation de puits domestiques ou agricoles a toujours existé, les habitants ne connaissent rien des nouvelles technologies de forage ( the new drilling machine »), qui permettent de creuser plus vite et plus profondément. Le nombre de rigs en activité est encore limité dans la région et leurs opérateurs proviennent majoritairement de l'état voisin, l'Andra Pradesh. Thaman voit dans le développement local d'un marché du captage d'eau une opportunité pour écouler des machines made in Tiruchengode. Pour les acteurs locaux du forage, l'enjeu commercial est de taille, il s'agit de leur proposer rapidement une technologie qui n'existe pas encore sur place. PRD est un assembleur de "petits lots », ce qui lui permet de faire du sur-mesure. Il ne s'agit pas de copier à l'identique mais d'améliorer l'existant. De la même manière que les carrosseries diffèrent d'une région à l'autre, le style des camions « tamouls » se distinguant de celui du Maharastra, les plateformes made in Tiruchengode s'inventent une forme propre. Une facture locale qui a son importance commerciale quand l'ensemble des entrepreneurs de forage du pays proviennent ou sont représentés dans la petite agglomération. Pour les constructeurs de Tiruchengode, nul besoin de démarcher pour trouver un débouché à leurs produits, le marché se trouve sur place, il se confond avec le district industriel attirant les clientèles de la filière. Vendeurs et acheteurs de machines de forage, se côtoient quotidiennement. En Inde, le marché du forage est gigantesque ${ }^{11}$.

\section{Un district monde de l'assemblage}

La croissance des activités d'assemblage et de forage distingue à l'échelle de l'Inde l'économie de Tiruchengode : "No other town can boast as deep a connection with the rest of the country as this little one in Tamil Nadu. Tiruchengode is the nation's borewell rig capital and thousands of machines and operators from here go down as much as 1,400 feet on any day, most months of the year ${ }^{12}$." Aujourd'hui, le district existe à côté des grands centres de production de la filière. Le métier des assembleurs locaux est reconnu par les entrepreneurs de forage de tout le pays, le marché de Tiruchengode attire les principaux équipementiers indiens ou étrangers de composants qui y installent leurs succursales. Au marché local de la fourniture de matériaux, de pièces détachées ou d'outillage s'agrègent les imposants magasins entrepôts des fabricants de composants. Tiruchengode tout comme la firme PRD s'accroît en développant des machines qui répondent aux besoins d'un marché en pleine croissance. PRD, à l'initiative de Paran, ingénieur en génie mécanique, petit-fils de forgeron, fils de tourneur, s'intéresse à tous les marchés hors de l'Union européenne et des États-Unis. Fabricant de petites séries, la firme familiale investit les niches et les marges du forage où les besoins sont immenses.

Les premiers contacts des entrepreneurs de Tiruchengode à l'étranger ont eu lieu avec des commerçants africains : " des Kényans sont venus nous voir, c'était en 1999 ». C'est tout le paradoxe des exportations de Tiruchengode. Ce ne sont pas les entreprises indiennes qui trouvent des débouchés à l'extérieur du sous-continent, mais des clientèles lointaines, en quête 

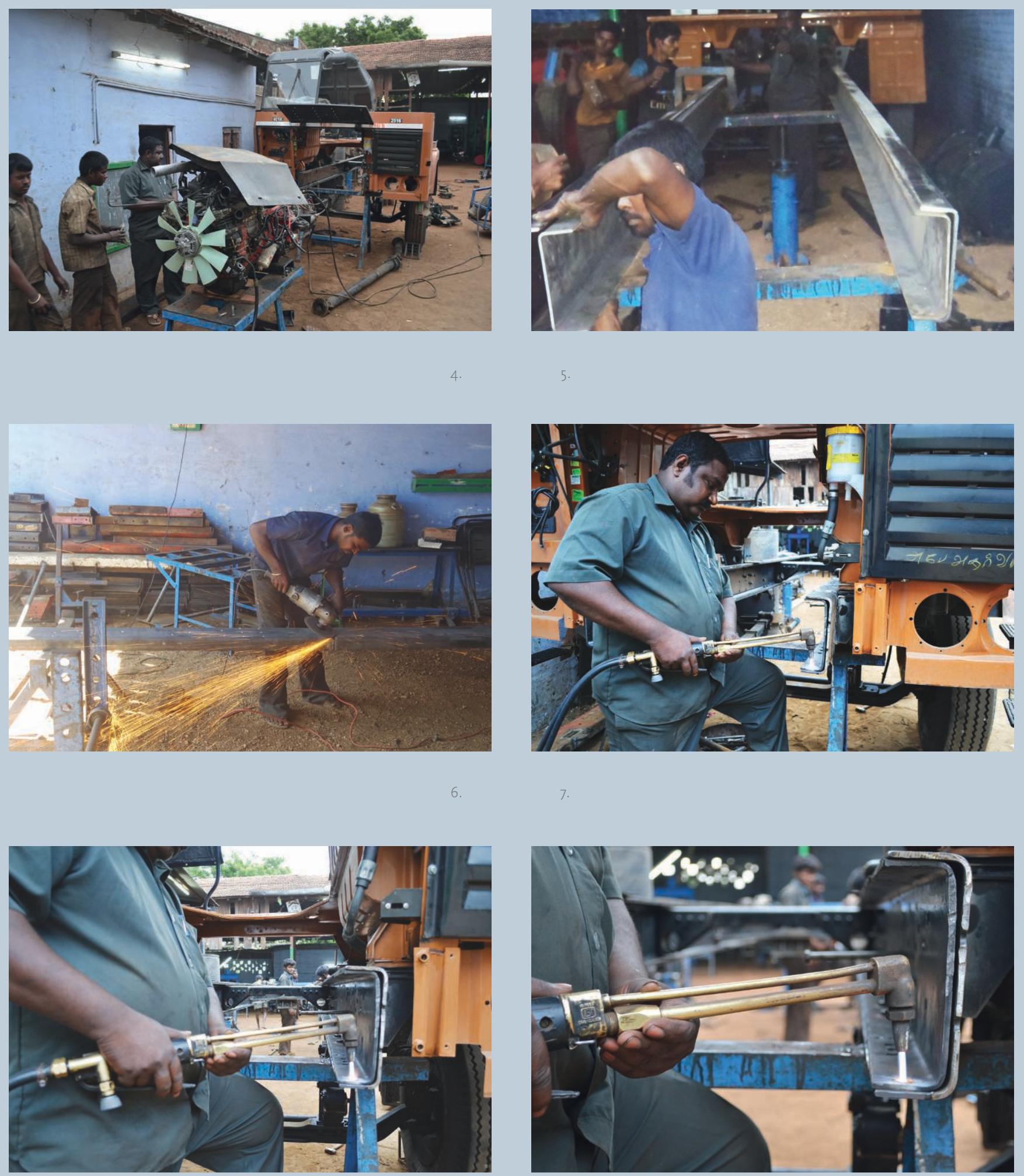

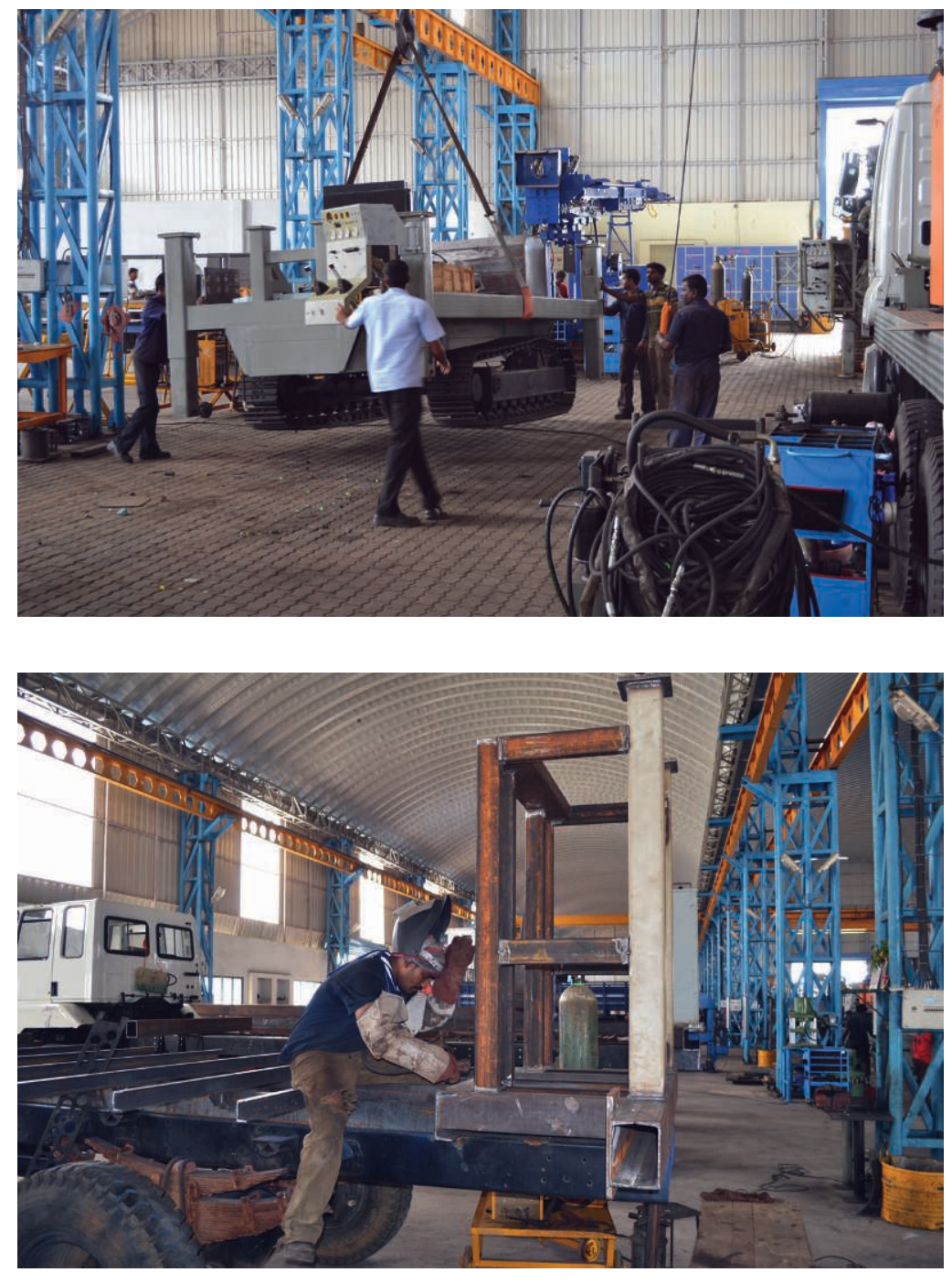

10 à 12. Nouvelle usine PRD de Perundura de fournisseurs qui découvrent le district périphérique ${ }^{13}$. PRD, qui n'avait jamais fait d'affaires ailleurs qu'en Inde, devient le pionnier de l'exportation à Tiruchengode grâce à la commande impromptue d'un entrepreneur kényan.

La firme assemble et livre rapidement des machines robustes, adaptées à des conditions difficiles d'exploitation et surtout faciles à maintenir en état de marche. La distribution de la technologie made in Tiruchengode s'appuie sur les formes de l'expertise technique les plus ordinaires. En effet, pour exister, être entretenus et réparés, les rigs peuvent compter sur d'innombrables petits ateliers autour du monde, et même dépendre de ses utilisateurs, pointant par là même, l'importance de la diffusion des compétences techniques. En Afrique, les ateliers délocalisés de PRD renouent avec les origines de l'entreprise familiale. Appartenant à une culture technique « do-it-yourself » de la maintenance, le meilleur moyen de promouvoir ces nouvelles machines est d'en assurer le service après-vente. Avec chaque plateforme vendue un technicien se déplace à demeure si nécessaire ( 1 an, 2 ans), pour former localement ses homologues. Au Ghana, PRD implante dans le cluster de Kumasi une succursale adossée à un atelier de maintenance.

Jordanie, Oman, Australie, Sri Lanka, Malaisie, Brésil, Mexique, l'entreprise PRD produit des biens matériels de niche destinés à des publics spécifiques dont les besoins sont négligés par la production en grandes séries. Des clientèles pauvres en quête de machines simples et résistantes, de technologies ouvertes et souples, c'est-à-dire intrinsèquement adaptables et réparables. Aujourd'hui, ce petit spécialiste accède aux marchés mondiaux pour diffuser les produits « sur mesure » de Tiruchengode et dispose de chaînes logistiques globales pour s'approvisionner en matériaux et composants. Pour cela, la production dédiée à l'exportation a été délocalisée en 2010 dans la Zone Économique Spéciale 
(Special Economic Zone) de Perundurai, à une quarantaine de kilomètres de Tiruchengode. La demande internationale contraint le groupe à changer d'échelle. PRD doit élargir physiquement son infrastructure d'assemblage. Mais la pression foncière dans son district historique condamne à moyen terme son développement lorsqu'à l'instigation des pouvoirs publics apparaissent de petites zones franches provinciales. C'est une aubaine pour quelques petites et moyennes entreprises exportatrices de la région tant les incitations sont fortes : disponibilité et prix des terrains, permis de construire, infrastructures, défiscalisation, équipements.

À Perundurai, la chaîne logistique s'ouvre aux petits :

"In SEZ, we can import anything duty free and we can export directly, there is not that much formality. We have some tax benefits. The only condition, you have to do export only, you cannot do local business. The main advantage, if you want to import anything, MAN Chassis or Mercedes Trucks from Germany, it is very easy. So I am able to give to international market what they want, whereas my competitor can give them only what is available in the local market. They can only give them Leyland or this kind of brand. But for me, if they ask me for a Mercedes, I can import a Mercedes at duty free. For import and export we have a green channel ${ }^{14}$.»

En Inde, sous l'influence des constructeurs japonais, les anciennes usines monolithiques sont remplacées par un écosystème de fournisseurs livrant des pièces en juste-à-temps. De plus en plus d'équipementiers étrangers s'installent. Les petites entreprises d'assemblage ne peuvent peut-être pas se fournir aux mêmes conditions de délais et de prix que les grands donneurs d'ordre, mais la chaîne logistique mondiale de l'automobile s'ouvre pratiquement à tous. Elle peut fonctionner par lots de plusieurs millions ou à l'unité : elle devient un réseau à « échelle libre $»$. Elle est aussi bien au service de petits entrepreneurs que de firmes émergentes. Dès lors, la chaîne de composition des rigs joue des logistiques locales et internationales. Les matériaux basiques, les composants low tech (comme le mât) arrivent de Tiruchengode, tandis que les éléments hydrauliques (control valve), les pièces les plus sophistiquées (high tech) proviennent désormais directement de fabricants européens (Italie, Allemagne) ou des États-Unis.

Le père et le fils peuvent enfin construire l'usine qu'ils imaginent depuis des années. À Perundurai, PRD trouve la place et l'infrastructure (il n'y a que très peu de coupures en électricité) qu'elle n'avait pas à Tiruchengode. C'est ici que la firme familiale achève l'intégration de l'ensemble de ses installations, ateliers, entrepôts, bureaux qu'elle a développés et
12. Des ateliers polyvalents dont la spécialisation souple permet de fabriquer à la demande une grande variété de machines

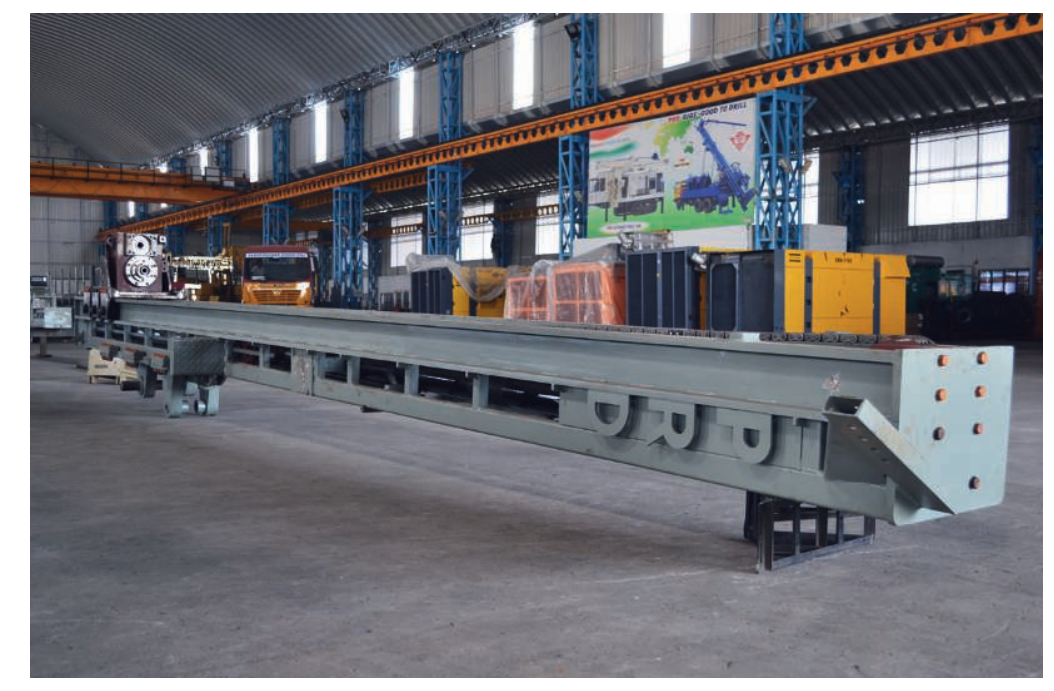


13. "Chaque ville est reconnue pour une chose en particulier, une spécialité. Cette ville est connue pour son savoir-faire en matière de forage. Tous les entrepreneurs en forage appartiennent à cette ville. Tu sais, j'ai beaucoup voyagé, et je n'ai jamais vu une telle concentration de rigs dans un si petit lieu. Quand les équipages reviennent à Tiruchengode pendant la mousson, on peut y trouver jusqu'à 3000 rigs », m'explique Paran (2013) des tâches qu'elle pouvait sous-traiter dans le district depuis une trentaine années. Paran et son père modernisent l'équipement (grue, bras de levage articulé, découpe numérique), ils organisent le stockage des pièces. Finalement, tout en conservant la manière de faire et d'assembler de Tiruchengode le nouvel immense hangar reconstitue un district miniature. Père et fils n'imaginent pas une chaîne d'assemblage intégrée mais des ateliers polyvalents dont la spécialisation souple permet de fabriquer à la demande une grande variété de machines, tout en se fournissant désormais dans le monde (cf. photos 10 à 12).

La polyvalence des ateliers de montage impressionne les fournisseurs italiens de composants hydrauliques de passage à Perundurai ${ }^{15}$, le catalogue aussi : engins d'exploration minière, de forage à grande dimension... Comme les rigs qu'ils fabriquent depuis maintenant une trentaine d'années, ces technologies de plus en plus complexes viennent d'ailleurs et ont été décomposées puis recomposées sur place. Si les clientèles du Kenya ou du Ghana viennent chercher à Tiruchengode des machines, bon marché et robustes, d'autres acheteurs lointains s'intéressent à la capacité indienne de refaire à bon prix et de bonne facture, des machines étrangères. PRD reproduit des biens matériels de niche destinés à des marchés délaissés. Et pas seulement sur le continent africain :

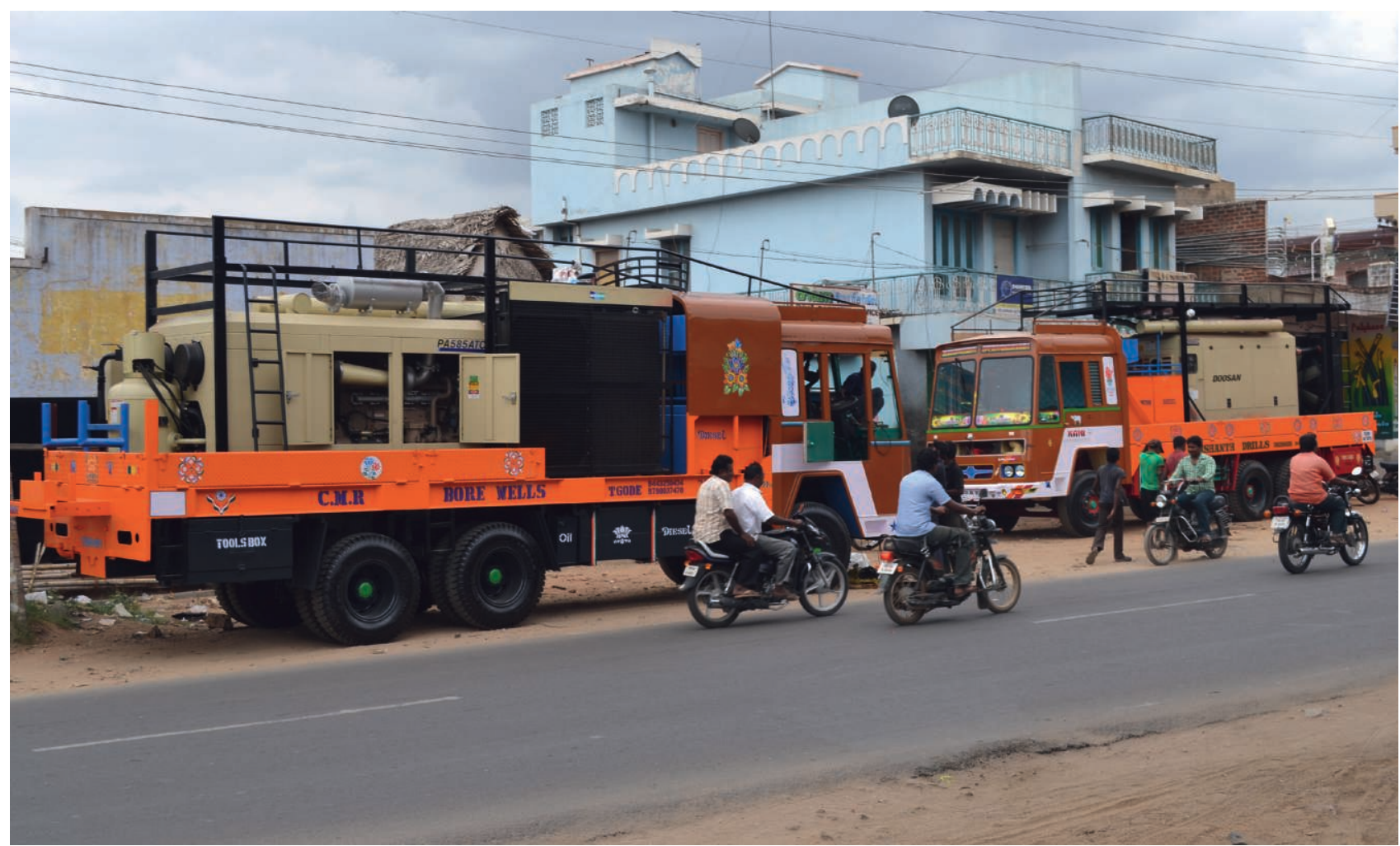


"The "Multi Star" is mining equipment used for the exploration. We had client from Australia, who was looking for a guy who could provide an Indian copy of that mining technology. The Australian client wanted an Indian manufacturer because this kind of machines were not made in Europe but only in the United-States. Europe has their own market, till recently they do not care about Australia. And when the Australians are going to United-States, they have to wait. Because the American manufacturers are providing for the entire world, so they are full. The delivery takes almost one year ${ }^{16}$. "

Des commanditaires australiens découvrent le district de Tiruchengode par l'intermédiaire de leurs contacts locaux. Ils sont à la recherche des fabricants indiens de rigs qui les exportent. L'exportation étant selon eux un critère de la qualité de la manière locale d'assembler. Les Australiens ne cherchent pas à Tiruchengode un produit mais un savoir-faire technique en matière d'assemblage. PRD ne peut concevoir sans avoir d'acheteur. Chaque projet de duplication optimise l'implication du demandeur. En plaçant de fait, une option d'achat sur le premier exemplaire, il finance le développement d'un prototype « grandeur nature » pour lequel le client fournit toute l'information technique nécessaire pour refaire cette machine, les plans, la liste des matériaux utilisés, (the guidelines, the inputs, the bills of materials). PRD a assemblé et vendu vingt-cinq exemplaires de ce modèle « Multi Star» dont le prix de revient est de l'ordre de 400000 \$. Des contrefaçons où se négocient concrètement des transferts technologiques, qui se jouent des systèmes des droits de propriété intellectuelle ( "brevets d'invention » ${ }^{17}$ ou « dessins et modèles ${ }^{18}$. Sur les enjeux économique et politique du brevet en Inde, voir le travaux de Guennif $\&$ Chaisse 2007). À chaque fois qu'il étend son réseau commercial, le groupe fait remonter de ces nouveaux marchés l'information dont il a besoin pour imiter les technologies en usage.

L'ethnographie des chaînes d'assemblages de Tiruchengode décrit un processus d'adaptation par rétro-ingénierie. Ainsi l'innovation s'appuie systématiquement sur ce que les autres ont déjà fait. Cette conception par la copie au cœur de la flexibilité industrielle des ateliers polyvalents du groupe PRD ${ }^{19}$, dont la fabrique subvertit le cycle classique du développement (besoin, design, implémentation, vérification, maintenance). C'est dans la maintenance de l'existant que simultanément se définissent les besoins, se conçoivent les prototypes (des contretypes seraient plus précis), que s'opère l'implémentation par de l'essai-erreur et l'expérimentation, de produits sur mesure. La firme ne conçoit rien ex nihilo, mais innove dans le processus de fabrication. En simplifiant les modèles qu'elle reproduit, elle gagne en productivité, en combinant des composants high tech (en provenance d'Italie par exemple) et low tech fabriqués dans le cluster, elle réalise des économies de coût à petite échelle. Cette forme semi-artisanale, semi-industrielle de la fabrication s'appuie en pratique, sans les nommer, sur des méthodes de conception agile ${ }^{20}$, propres au mouvement DIY (Do-It-Yourself), et qui caractérisent de nouvelles formes de production. Ces "self made engineers ", fils d'anciens forgerons indiens devenus des assembleurs d'appareils mobiles de forage, exportent principalement une technologie ouverte, modifiable, elle-même réversible ou braconnable par ses utilisateurs.

Adaptation et expérimentation permanentes s'émancipent des règles bien établies et défient les grands acteurs du secteur. PRD n'est pas un simple fabricant de produits contrefaits, le groupe développe un vrai esprit d'innovation avec des possibilités d'évolution répondant 
14. Après avoir été copié, le modèle Multistar est ajouté au catalogue de PRD. Son prix de vente : $400000 \$$ aux normes mondiales. En Afrique, PRD capture de nouveaux marchés dont les besoins sont proches de son marché d'origine. L'entreprise indienne cherche des débouchés peu prospectés dans le forage de puits d'eau, parce que jugés peu rentables par les grands industriels (USA, UE, Australie). Des initiatives modestes où l'adaptation créative de techniques exogènes et lointaines est dictée par la contrainte économique. Échecs, essais, compromis, scandent l'introduction des camions de seconde main, façonnent ses composants, détournent l'objet en action. Dans les ateliers de maintenance du Kenya ou du Ghana, les châssis de camions allemands et leurs plateformes indiennes sont retravaillés, remodelés, reconstruits. Les mécaniciens « s'arrangent avec les moyens du bord », combinent le neuf et le « déjà là », pour créer des matériels appropriés à partir de pièces standards modifiées. Les mécaniciens défendent par leur activité un projet contre entropique (l'entropie serait la dégradation irréversible de toute chose, « sans moyen de recoller les morceaux »), où les produits manufacturés sont doués d'une force gestative. Refabriquer, c'est donc réparer et restaurer, mais c'est aussi « faire à nouveau ». Entre leurs mains réparatrices, les objets ont d'abord une capacité à se régénérer. Contre l'idée d'« obsolescence programmée » des grands constructeurs, un autre modèle se fait jour, celui d'une « éternité non programmée des techniques $»$, dans cette interaction entre les ingénieurs indiens qui réinventent les technologies et les fitters (monteurs ou installateurs) ghanéens qui font tout pour les prolonger le plus longtemps possible.
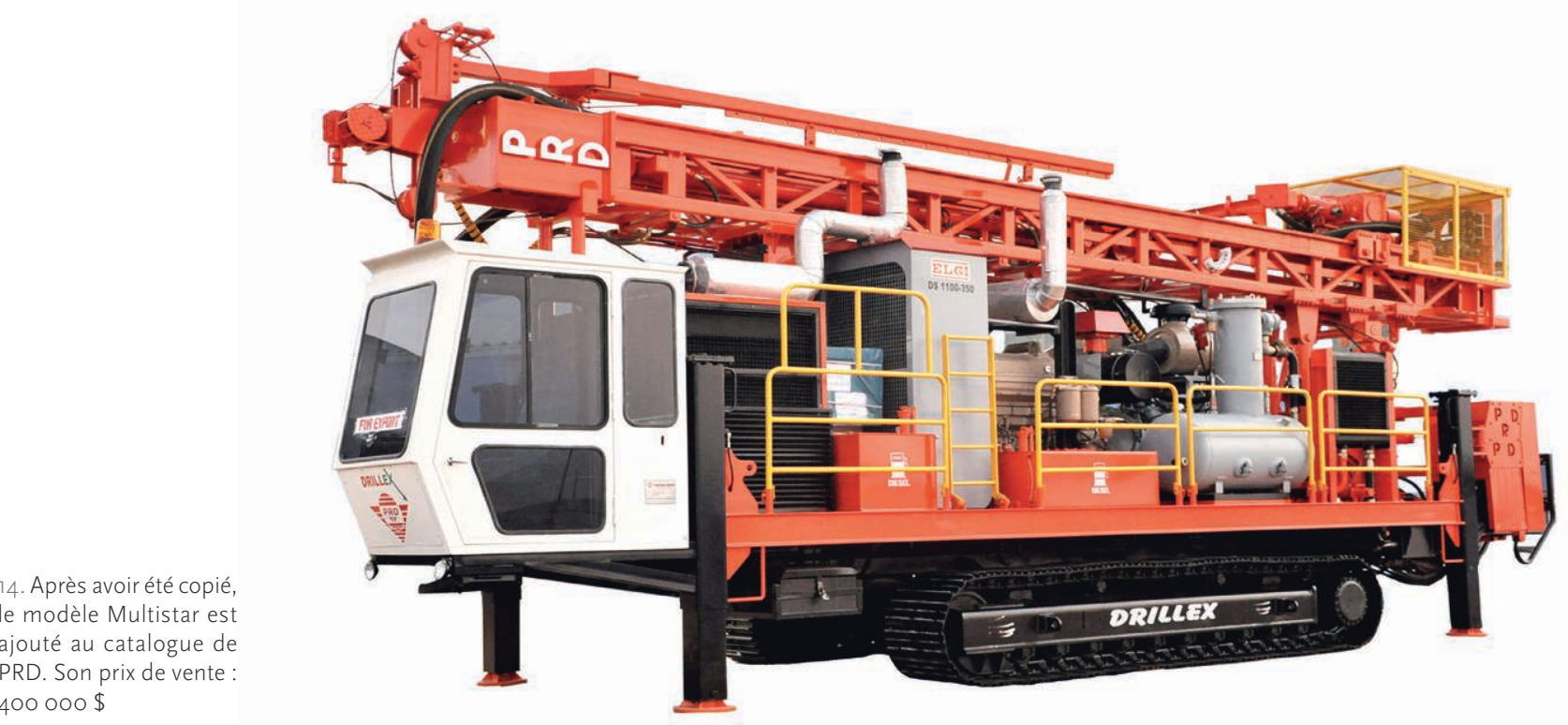


\section{Notes}

1. Cf. le cas de l'industrie japonaise du cycle (Edgerton $2007: 142)$

2. «A small textile town in the Salem-Coimbatore cotton-weaving belt » (Cadène 1998).

3. Un pont moteur est un système de suspension de véhicule à essieu rigide dans lequel les arbres de transmission qui fournissent la puissance motrice aux roues servent à relier celles-ci latéralement de sorte qu'elles tournent en permanence dans le même axe.

4. Entretien avec M. T. P. Thangaraj (alias Thaman), Chairman et fondateur de PRD groups.

5. Génie mécanique vs génie civil : sur route, l'usure du revêtement augmente très rapidement avec celle de la charge à l'essieu. Ainsi un poids lourd chargé à 10 tonnes par essieu use la route 10000 fois plus qu'une voiture à 1 tonne par essieu.

6. Entretien avec M. T.P. Thangaraj (alias Thaman).

7. À propos de la Tamil Nadu Lorry Body Builder Federation, voir les travaux de Raman (2013).

8. Autobus, camions, camionnettes Bedford assemblés à Luton (UK) ont été massivement exportés dans les anciennes colonies de l'empire britannique. Ainsi, le modèle de camionnettes Bedford CA (1952-1969) apparait conjointement sur les routes et dans les récits indiens, égyptiens ou ghanéens des pionniers de la mécanique et du transport. Neufs ou anciens, ils étaient importés finis ou en pièces pour être assemblés sur place (comme à Tema au Ghana, où trotros et Mammy lorries sont montés jusqu'en 1966). Il est intéressant de noter que les artisans des colonies anglaises ont fait leur apprentissage mécanique sur les premières générations de modèles identiques.

9. Entretien avec Paranthaman (alias Paran), fils de Thaman.

10. À propos de l'organisation du travail au sein du district et des coûts de fabrication, voir les travaux de Cadène op. cit. et Tastevin 2017.

11. «In India 53\% of irrigation water is supplied from groundwater. The groundwater also accounts for over four-fifth of drinking water in India » : Cf. http://www.indiaenvironmentportal.org.in/files/file/Groundwater\%20Year\%20 Book\%202012-13.pdf ou encore http://www.nih.ernet. in/rbis/india_information/groundwater.htm.

12. The Hindu, July 28, 2013.

13. Tout comme l'autorickshaw de Bajaj qui arrive finalement pour la première fois en Afrique dans les petites villes du delta du Nil, à l'instigation d'entrepreneurs égyptiens. (cf. Tastevin 2015 « Bajaj en Égypte ou la diffusion de l'autorickshaw en Afrique », Autrepart 76).

14. Entretien avec Paran.

15. Depuis maintenant une vingtaine d'années, les fabricants italiens de composants hydrauliques, de petites industries familiales de la région de Pérouse, ont leurs représentants en Inde. Des intermédiaires originaires, le plus souvent de Bombay, prospectent pour le compte de leur client étranger le marché local. Ce jour-là, le fils de l'un de ces patrons italiens visite en compagnie de son représentant indien, les installations de PRD, qui renégocie directement, fort des capacités nouvelles de cette usine, les achats de composants.

16. Entretien avec Paran.

17. Le « brevet d'invention » protège une innovation technique, c'est-à-dire un produit ou un procédé qui apporte une nouvelle solution technique et susceptible d'application industrielle. La loi sur les brevets (Patent Act de 1970, amendé en 2005) précise de manière quasi exhaustive les inventions non brevetables en Inde. Sont ainsi exclus de la brevetabilité : les inventions « frivoles », contraires à l'ordre public et aux bonnes mœurs, les méthodes diagnostiques, thérapeutiques et chirurgicales, les végétaux et animaux, programmes informatiques et mathématiques, sauf intégrés à du matériel. (cf. La Propriété Intellectuelle en Inde, INPI).

18. Pour être enregistré, le « dessin » ou « modèle » doit avoir une forme, couleur ou apparence spécifique, être nouveau et être utilisé dans l'industrie. L'Inde s'est conformée aux règles internationales d'enregistrement et de protection dans le cadre du New Design Act de 2000 (cf. La Propriété Intellectuelle en Inde, INPI).

19. Mais c'est le cas ailleurs comme en Chine pour l'électronique près de Shen Zhen, dans le delta de la rivière des Perles, où s'est développé un écosystème de la contrefaçon appelé Shan Zhai.

20. Depuis une quinzaine d'années, la majorité des développements de logiciels s'appuie sur des méthodes dites « agiles ». Sous cette bannière se regroupent plusieurs méthodes basées sur un développement itératif et incrémental, dans lequel la recherche de solutions aux problèmes rencontrés s'appuie sur la collaboration de pair à pair. Elle promeut des réponses rapides et flexibles, une planification des tâches adaptatives dans des laps de 
temps très courts permettant une très grande réactivité. L'objectif central est de trouver de meilleurs moyens de développer des logiciels. Cet ensemble de méthodes a fait ses preuves dans le domaine du logiciel libre et pourrait pour certains s'appliquer aux méthodes de travail de la production de biens.

\section{I'auteur}

Yann Philippe Tastevin est anthropologue (LISST, CNRS-Université de Toulouse, France). Ses travaux portent sur les processus d'innovation au Sud et les circulations globalisées des technologies. Ils explorent l'évolution des technologies de transport (rickshaw, tacots, camions) hors de l'espace et du temps où celles-ci ont été produites. Ces technologies réinventées dans des ateliers polyvalents, assemblées sur des chaînes de montage à ciel ouvert, entretenues au coin de la rue par des artisans, des ingénieurs, des mécaniciens circulent dans le monde entier à l'initiative d'entrepreneurs transnationaux. Tout l'enjeu d'une démarche résolument ethnographique est alors de combiner une anthropologie des techniques et une anthropologie économique des dynamiques commerciales transnationales qui sous-tendent les logiques d'usage de ces technologies. Yann Philippe Tastevin achève la rédaction d'Autorickshaw : émergence et recomposition d'une filière entre l'Inde, l'Égypte et le Congo, à paraître aux éditions Karthala, collection « Terre et gens d'Islam ».

\section{Iconographie}

Image d'ouverture. Forage d'un puits dans une maison nouvellement construite. Il s'agit d'un testeur grandeur nature d'une plateforme mobile (rigs) qui doit être exportée au Nigéria. (C) Yann Philippe Tastevin.

\section{Références}

Cadène, P. 1991 « Urban Configurations and Merchant Networks in South India. A workshop », Pondy Papers in Social Sciences 9.

Cadène, P. \& M. Holmström 1998 Decentralized Production in India. Dehli : Sage Publications.

Edgerton, D. 2007 [2013] The Shock of the Old: Technology and Global History Since 1900. Oxford University Press. [Traduction française. Paris : Seuil].

Guennif, S. \& J. Chaisse 2007 « L'économie politique du brevet au Sud : variations indiennes sur le brevet pharmaceutique », Revue Internationale de Droit Economique XXI (2).

Kundu, A. 1994 «Industrial Development, Its Dispersal and Integration of Medium Sized Towns in the National Economy: the Case of Tiruchengode in Tamil Nadu », Indian Journal of Industrial Relations $29: 321-334$.

\section{Pour citer l'article}

Tastevin, Y. P. 2017 «Des chars à bœufs aux plateformes mobiles de forage. Réparation, fabrication et innovation à Tiruchengode», TechniquesECulture 67 « Low tech? Wild tech! », p. 196-211.
Crédits photographiques pour l'ensemble des images: (C) Yann Philippe Tastevin sauf :

1. Sources : Roda, J.-M. 1994.

2. Sources: Archives personnelles de Thaman (1980).

Raman, B. 2014 « Patterns and Practices of Spatial Transformation in Non-Metros: The Case of Tiruchengode », Review of Urban Affairs, Economic \& Political Weekly XLIX (22).

- 2013 « The Logics and Realms of Small Town Territory. The Story of Tiruchengode », SARAI Reader 9 : 200-210.

Roda, J.-M. 1994 Les filières du bois en Inde du Sud. Le cas de Tiruchengode, Tamil Nadu. Mémoire de fin d'études, sous la direction de Philippe Cadène. Université de Bordeaux.

Tastevin, Y. P. 2015 « Bajaj en Égypte ou la diffusion de l'autorickshaw en Afrique », Autrepart 76.

Tastevin, Y. P. 2017 « Ox Carts and Borewell Rigs: From Maintenance to Manufacture and Innovation in Tiruchengode» in E. Denis \& M.-H. Zehra dir. Subaltern Urbanization in India. New Delhi : Springer. The Hindu 2013 Sunday, July 28 « The deep water crisis». 
